\title{
The Photon Wave Function
}

\author{
Joseph Cugnon \\ AGO Department, University of Liège, allée du 6 Août 17, bât. B5, B-4000 Liège 1, Belgium \\ E-mail:cugnon@plasma.theo.phys.ulg.ac.be
}

Received September 13, 2011; revised October 15, 2011; accepted October 23, 2011

\begin{abstract}
The properties of a wave equation for a six-component wave function of a photon are re-analyzed. It is shown that the wave equation presents all the properties required by quantum mechanics, except for the ones that are linked with the definition of the position operator. The situation is contrasted with the threecomponent formulation based on the Riemann-Silberstein wave function. The inconsistency of the latter with the principles of quantum mechanics is shown to arise from the usual interpretation of the wave function. Finally, the Lorentz invariance of the six-component wave equation is demonstrated explicitly for Lorentz boosts and space inversion.
\end{abstract}

Keywords: Photon, Wave Function, Quantum Mechanics, Lorentz Invariance

\section{Introduction}

The wave function of a photon is a topic that has for long been ignored since the physicists have been primarily interested in emission and absorption processes, for which solid theories, such as the Glauber theory, exist. But in the last two decades, an increased interest has been focused on the description of a single photon by a wave function. Two difficulties have shown up. The first one deals with the problem of the proper wave equation. The second one is linked with the definition of the position operator. Largely irrespective of the proper wave equation, it seems difficult to define a position operator as usual, which, at the same time, satisfies the commutation relations with the total angular momentum dictated by Poincaré symmetry. It should however be mentioned that acceptable wave functions have been shown to describe a photon with good localization properties.

In this paper, we focus on the first question mentioned above, namely the wave equation. It has been realized during the last years, that the wave equation should be similar, or at least consistent, with the Maxwell equations. But the proper form of the wave equation is still controversial. Furthermore, for some choices, the wave function does not fulfill basic principles of quantum mechanics. Here, we study a particular wave equation and show it to be consistent with the requirements of quantum mechanics and to present good symmetry properties. It nevertheless presents the same problems as regarding the definition of the position operator.

\section{The Wave Equation}

\subsection{Introduction}

The systematic search for a relativistic wave equation for the photon has been undertaken by several authors and in particular in References [1-7], to restrict oneself to the recent years. Without entering into details, the wave equation should be linear in the time derivative and assume the canonical form

$$
\frac{\partial \psi}{\partial t}=-\frac{i}{\hbar} H \psi
$$

where the Hamiltonian $H$ is linear in the momentum $p$ and is such that plane wave solutions are consistent with the relativistic dispersion relation. More precisely, $H$ should be a square root of the operator $p^{2} c^{2}$ in the space of the components, like the Dirac Hamiltonian is a square root of the operator $p^{2} c^{2}+m^{2} c^{4}$ in the space of the Dirac spinors. On the other hand, the number of components of $\psi$ is not fixed a priori. It is reasonable to admit that it should be chosen as low as possible, for the sake of simplicity.

As a guide to determine the minimum number of components, it is instructive to look at other simple examples. For massive boson particles with spin zero, $\psi$ has two components and $H$ can be taken as 


$$
H=\left(\begin{array}{cc}
-\frac{p^{2}}{2 m}-m c^{2} & -\frac{p^{2}}{2 m} \\
\frac{p^{2}}{2 m} & \frac{p^{2}}{2 m}-m c^{2}
\end{array}\right)
$$

This is the well-known two-component formulation of the Klein-Gordon equation [8], linear in the time derivative. The $m=0$ limit is not very much instructive, since it is singular. On the other hand, this equation suggests that an equation for a spin one boson should have more than two components. For a massive spin $1 / 2$ particle, $\psi$ has four components and $H$ has the Dirac form

$$
H_{D}=\boldsymbol{\alpha} \cdot \boldsymbol{p} c+\beta m c^{2} .
$$

Although it applies to fermions, this equation strongly suggests that the Hamiltonian $\mathrm{H}$ of Equation (1) should be taken as a linear and homogeneous form in the momentum $p$. In the $m=0$ limit, the Dirac equation reduces to the Weyl equation, which shows that the appropriate number of components for massless spin $1 / 2$ particles is two. So, again, the minimum number of components for the photon wave function seems to be three. This choice have been made by several authors. The form of $H$ is then practically uniquely determined and the equation is:

$$
\frac{\partial \psi}{\partial t}=-\frac{i}{\hbar} i c \boldsymbol{p} \times \boldsymbol{\psi}
$$

The Hamiltonian $H=i c \boldsymbol{p} \times$ is hermitian because the operator $p \times=-i \hbar \nabla \times$ is anti-hermitian. This can be seen from the following property:

$$
\nabla \times \boldsymbol{\psi}=\sum_{k} \frac{\partial}{\partial x_{k}}\left(\boldsymbol{H}_{\boldsymbol{k}}\right) \cdot \boldsymbol{\psi}=\frac{i}{\hbar} \sum_{k} p_{k}\left(\boldsymbol{H}_{\boldsymbol{k}}\right) \cdot \boldsymbol{\psi}
$$

where the matrices $\boldsymbol{H}_{\boldsymbol{k}}$ are given explicitly by

$$
\boldsymbol{H}_{1}=\left(\begin{array}{ccc}
0 & 0 & 0 \\
0 & 0 & -1 \\
0 & 1 & 0
\end{array}\right), \boldsymbol{H}_{2}=\left(\begin{array}{ccc}
0 & 0 & 1 \\
0 & 0 & 0 \\
-1 & 0 & 0
\end{array}\right), \boldsymbol{H}_{3}=\left(\begin{array}{ccc}
0 & -1 & 0 \\
1 & 0 & 0 \\
0 & 0 & 0
\end{array}\right)
$$

See Appendix A for details. In the following, we will use the short-hand notation

$$
\boldsymbol{p} \cdot \boldsymbol{H}=\sum_{i} p_{i}\left(H_{i}\right)
$$

the presence of $\boldsymbol{H}$ indicating automatically $3 \times 3$ matrices in the space of the components.

The wave Equation (4) is compatible with the criteria enunciated above, namely that the square of $\boldsymbol{H}$ is equal to $p^{2} c^{2}$. This however requires some restriction on the wave functions. Indeed, the square of the Hamiltonian matrix is given, with the help Equation (64) of Appendix A, by

$$
(i c \boldsymbol{p} \cdot \boldsymbol{H})^{2}=\left(i c \sum_{i} p_{i}\left(H_{i}\right)\right)^{2}=p^{2} c^{2} \underline{1}-c^{2} \boldsymbol{p} \otimes \boldsymbol{p}
$$

where 1 is the $3 \times 3$ unit matrix and where the second term is the direct product of $\boldsymbol{p}$ by itself. This operator reduces to the $p^{2} c^{2}$ operator, if the wave functions are restricted to divergenceless functions, satisfying $\nabla \cdot \boldsymbol{\psi}=0$ or, equivalently, $\boldsymbol{p} \cdot \boldsymbol{\psi}=0$.

Equation (4) corresponds to the Maxwell equations, as motivated and discussed below. However, this is only true for a special and criticizable choice of the wave function, as explained later.

\subsection{An Equation with Good Quantum Mechanical Features}

The proposed equation involves a six component wave function that we will write as

$$
\psi=\left(\begin{array}{l}
\Phi_{1} \\
\Phi_{2}
\end{array}\right),
$$

$\Phi_{1}, \Phi_{2}$ both having three components and being divergenceless. The wave equation is given by:

$$
\frac{\partial}{\partial t}\left(\begin{array}{l}
\Phi_{1} \\
\Phi_{2}
\end{array}\right)=-\frac{i}{\hbar}\left(\begin{array}{cc}
0 & -c \boldsymbol{p} \cdot \boldsymbol{H} \\
c \boldsymbol{p} \cdot \boldsymbol{H} & 0
\end{array}\right)\left(\begin{array}{l}
\Phi_{1} \\
\boldsymbol{\Phi}_{2}
\end{array}\right),
$$

where all elements of the matrix on the right-hand side are $3 \times 3$ matrices. The non-vanishing $3 \times 3$ matrices are anti-hermitian, but the big $6 \times 6$ matrix, which is nothing but the Hamiltonian, is hermitian. It is hermitian in the space of the components and in the space of the normalizable functions $\Phi_{1}$ and $\Phi_{2}$, due to the presence of the momentum operator. Equation (10) has also been proposed by Wang et al. [9,10], Bialynicki-Birula [3] and others ${ }^{1}$. Here we study in some detail the quantum and symmetry properties of this wave equation.

\subsection{Quantum Properties}

We are going to show that Equation (10) has good quantum mechanical properties, except for the ones that are linked with the position operator.

1) Hilbert space. Like for Equation (4), it is necessary to restrict the functions $\Phi_{1}$ and $\Phi_{2}$ to be divergenceless in order to ensure $H^{2}=p^{2} c^{2}$. This is easily verified using Equation (8). The total configurational Hilbert space is thus the direct product of two similar Hilbert spaces built on normalizable divergenceless functions in configurational space. These are perfect Hilbert spaces. A possible (limiting) basis is provided by familiar plane waves with transverse polarization.

${ }^{1}$ E. Majorana seems to have proposed this choice for the first time [11]. 
2) Probability density and current. It is easy to check, by the usual method, that one can define a probability density

$$
\rho=\left|\Phi_{1}\right|^{2}+\left|\Phi_{2}\right|^{2}
$$

and a probability current

$$
\boldsymbol{j}=2 \mathfrak{R}\left(c \Phi_{1} \times \Phi_{2}^{*}\right)
$$

which satisfy the continuity equation. Details are given in Appendix B.

3) Phase of the wave function. If $\psi$ given by Equation (9) is a solution of Equation (10), then

$$
\psi^{\prime}=\psi e^{i \phi}
$$

where $\phi$ is a real constant, is also a solution. This results from the linearity of the equation. Transformation (13) leaves the density and current of probability invariant, as it should. It is of interest to notice that the wave equation (10) is real, in the sense that, besides the functions $\Phi_{1}$ and $\Phi_{2}$, it involves real operators and real coefficients. Indeed, owing to Equation (5), it can be written as

$$
\begin{aligned}
& \frac{1}{c} \frac{\partial}{\partial t} \Phi_{1}=\nabla \times \Phi_{2} \\
& \frac{1}{c} \frac{\partial}{\partial t} \Phi_{2}=-\nabla \times \Phi_{1}
\end{aligned}
$$

The other remarkable property, which is shared by the Weyl equation, is that $\hbar$ has disappeared.

4) Plane wave solutions and energy eigenvalues. Let us consider solutions of Equation (10) of the type

$$
\psi=a e^{i k . x}=a e^{i(\boldsymbol{k} . \boldsymbol{r}-\omega t)},
$$

where $a=\left(\begin{array}{l}\boldsymbol{a}_{1} \\ \boldsymbol{a}_{2}\end{array}\right)$ is a six-component column vector,

with both $\boldsymbol{a}_{1}$ and $\boldsymbol{a}_{2}$ orthogonal to $\boldsymbol{k}$. Inserting form (15) into Equation (10), one gets the eigenvalue problem expressed by

$$
\left(\begin{array}{cc}
\omega & -c \boldsymbol{k} \cdot \boldsymbol{H} \\
c \boldsymbol{k} \cdot \boldsymbol{H} & \omega
\end{array}\right)(a)=0 .
$$

The eigenvalues are $\omega=0, k c,-k c$, with $k=|\boldsymbol{k}|$, each doubly degenerate. A set of eigenvectors can be constructed as follows. Let us define

$$
\varepsilon_{L}=\frac{\boldsymbol{k}}{k}=\boldsymbol{k}^{0},
$$

$\boldsymbol{\varepsilon}_{1}$ any real unit vector orthogonal to $\boldsymbol{k}$

$$
\boldsymbol{\varepsilon}_{1} \cdot \boldsymbol{k}^{0}=0, \quad \boldsymbol{\varepsilon}_{1} \cdot \boldsymbol{\varepsilon}_{1}=1
$$

and

$$
\boldsymbol{\varepsilon}_{2}=\boldsymbol{k}^{0} \times \boldsymbol{\varepsilon}_{1} .
$$

The eigenvectors can taken as:

$$
\begin{aligned}
& \omega=0: \cdots a=\left(\begin{array}{c}
\boldsymbol{\varepsilon}_{L} \\
\boldsymbol{\varepsilon}_{L}
\end{array}\right), \cdots a=\left(\begin{array}{c}
\boldsymbol{\varepsilon}_{L} \\
-\boldsymbol{\varepsilon}_{L}
\end{array}\right), \\
& \omega=k c: \cdots a=\left(\begin{array}{c}
\boldsymbol{\varepsilon}_{1} \\
\boldsymbol{\varepsilon}_{2}
\end{array}\right), \cdots a=\left(\begin{array}{c}
\boldsymbol{\varepsilon}_{2} \\
-\boldsymbol{\varepsilon}_{1}
\end{array}\right), \\
& \omega=-k c: \cdots a=\left(\begin{array}{c}
\boldsymbol{\varepsilon}_{1} \\
-\boldsymbol{\varepsilon}_{2}
\end{array}\right), \cdots a=\left(\begin{array}{c}
\boldsymbol{\varepsilon}_{2} \\
\boldsymbol{\varepsilon}_{1}
\end{array}\right) .
\end{aligned}
$$

This is easily verified using Equation (10). The eigenvectors for $\omega=0$ are just formal solutions of Equation (16). If one requires these eigenvectors to be orthogonal to $\boldsymbol{k}$, there is simply no eigenvector. This result corresponds to the fact that the photon cannot have a longitudinal polarization. The positive eigenvalues correspond to two independent transverse polarizations. It is easy to check that the various solutions are orthogonal to each other. The negative eigenvalues correspond to propagation in the opposite direction. In general, negative eigenvalues are associated with antiparticles. The fact that negative eigenvalues solutions simply duplicate the positive ones correspond to the fact the photon is identical to its antiparticle or, equivalently, has no antiparticle.

Alternatively, one can consider circular polarization. A set of eigenvectors is then provided, for the $\omega= \pm k c$ eigenvalues, by

$$
\begin{gathered}
\omega=k c \cdot \cdots a=\left(\begin{array}{c}
\varepsilon^{+} \\
-i \varepsilon^{+}
\end{array}\right), \cdots a=\left(\begin{array}{c}
\varepsilon^{-} \\
i \varepsilon^{-}
\end{array}\right) \\
\omega=-k c \cdot \cdots a=\left(\begin{array}{c}
\varepsilon^{+} \\
i \varepsilon^{+}
\end{array}\right), \cdots a=\left(\begin{array}{c}
\varepsilon^{-} \\
-i \varepsilon^{-}
\end{array}\right)
\end{gathered}
$$

with

$$
\varepsilon^{ \pm}=\varepsilon_{1} \pm \varepsilon_{2}
$$

The orthogonality between different solutions $a_{i}$ and $a_{j}$ is to be understood in the sense of the following scalar product $\left(a_{i}, a_{j}\right)=a_{i}^{*} \cdot a_{j}=\delta_{i j}$.

5) Spin and helicity operators. The spin operator can be taken as

$$
\boldsymbol{S}=\hbar\left(\begin{array}{cc}
i \boldsymbol{H} & 0 \\
0 & i \boldsymbol{H}
\end{array}\right)
$$

We remind that the $H_{k}$ 's are antihermitian operators. Actually, the operators $i H_{k}$ form the adjoint representation of the SU(2) algebra (see Equation (71)) and are thus the natural representation for spin one (3 components). Like for the Dirac equation, the operator $\boldsymbol{S}$ does not commute with the Hamiltonian in Equation (10), but $\boldsymbol{J}=\boldsymbol{L}+\boldsymbol{S}$ does, as it can be easily verified $(\boldsymbol{L}=\boldsymbol{r} \times \boldsymbol{p})$. 
Actually, one has

$$
[H, \boldsymbol{L}]=-[H, \boldsymbol{S}]=-i c \hbar \times \boldsymbol{\alpha}
$$

with

$$
\alpha=\left(\begin{array}{cc}
0 & -\boldsymbol{H} \\
\boldsymbol{H} & 0
\end{array}\right) .
$$

The operator $S^{2}$, which is equal to $2 \hbar^{2}$, owing to Equation (69), obviously commutes with the Hamiltonian.

The operator

$$
\boldsymbol{p} \cdot \boldsymbol{S}=\hbar=\left(\begin{array}{cc}
i \boldsymbol{p} \cdot \boldsymbol{H} & 0 \\
0 & i \boldsymbol{p} \cdot \boldsymbol{H}
\end{array}\right)
$$

commutes with the Hamiltonian, as well as with the momentum operator $\boldsymbol{p}$. Therefore the eigenvectors of $H$ can be taken as eigenvectors of $\boldsymbol{p}$ and of the helicity operator

$$
h=\frac{\boldsymbol{p} \cdot \boldsymbol{S}}{\hbar \sqrt{\boldsymbol{p} \cdot \boldsymbol{p}}} .
$$

Actually, this is case for the eigenvectors of $H$ built on the vectors of Equation (23). They are eigenvectors of the helicity operator with eigenvalues $h=1,-1,1,-1$, respectively. Here again, the negative eigenvalue solutions duplicate the positive ones.

\subsection{The Velocity “Operator"}

Let us consider the velocity "operator" defined by the relation

$$
\frac{\mathrm{d}}{\mathrm{d} t}\langle\boldsymbol{r}\rangle=\frac{i}{\hbar}\langle[H, \boldsymbol{r}]\rangle .
$$

For the Hamiltonian defined in the wave Equation (10), one has

$$
\frac{\mathrm{d}}{\mathrm{d} t}\langle\boldsymbol{r}\rangle=c\left\langle\begin{array}{cc}
0 & -\boldsymbol{H} \\
\boldsymbol{H} & 0
\end{array}\right\rangle=\boldsymbol{\alpha},
$$

where $\alpha$ is given by Equation (27), which tells that $\alpha$ is the velocity operator in units of $c$. The eigenvalues of the operators $\alpha_{i}, i=1,2,3$, are equal to 0 (with no divergenceless eigenfunctions) and \pm 1 . The eigenfunctions are the same as in Equations $(21,22)$ (or as in Equation (23) for the \pm 1 energy eigenvalues, as can be checked directly, despite the fact that $\alpha$ and $H$ do not commute. This strange property is also related to the fact that the $\alpha_{i}$ operators do not commute and therefore one cannot build an eigenstate of $\alpha_{1}$ which is an eigenstate of $\alpha_{2}$ at the same time. In other words, a precise measurement of the x-component of the velocity is incompatible with a precise measurement of the y-component. A similar di- fficulty exists for the Dirac equation. Incidentally, we mention that, like for the Dirac equation, Equation (31) is equivalent to

$$
\frac{\mathrm{d}}{\mathrm{d} t}\langle\boldsymbol{r}\rangle=\int j \mathrm{~d}^{3} \boldsymbol{r},
$$

where $\boldsymbol{j}$ is the probability current defined by Equation (12). We will not elaborate any further on this point, which is beyond the scope of this note.

These difficulties are linked with the non-existence of the position operator for the photon. There is a large literature on this point (see [3] for a review). Heuristically, the non-existence of the position operator derives simply from the fact that the multiplication by $\boldsymbol{r}$ of a divergenceless function does not yield a divergenceless function. Therefore the position operator has no eigenvalue.

\subsection{Interpretation of the Wave Function}

It is often argued that if the photon can be viewed as "an elementary excitation of the quantized electromagnetic field" and as a particle at the same time, its wave function should be related to the (average) electric and magnetic fields that it carries, i.e. with physical electromagnetic fields.

We discuss this relationship first for the wave Equation (4). We will closely follow here the arguments of Reference [12]. The three-component Equation (4) may be written

$$
\frac{\partial \psi}{\partial t}=-i c \nabla \times \psi
$$

Assuming

$$
\boldsymbol{\psi}=\boldsymbol{E}+i \boldsymbol{B}
$$

where $\boldsymbol{E}$ and $\boldsymbol{B}$ are real functions ${ }^{2}$, one gets after substitution and separation of real and imaginary parts,

$$
\begin{aligned}
& \frac{1}{c} \frac{\partial \boldsymbol{E}}{\partial t}=\nabla \times \boldsymbol{B}, \\
& \frac{1}{c} \frac{\partial \boldsymbol{B}}{\partial t}=-\nabla \times \boldsymbol{E},
\end{aligned}
$$

which are nothing but the Maxwell equations for free transverse fields. According to the authors of Reference [12], the Maxwell equations provide a "correct relativistic, quantum theory of the light quantum". It is thus tempting to interpret, as did the authors of this work, $\boldsymbol{E}$ and $\boldsymbol{B}$ as the electric and magnetic fields. This interpretation raises a certain number of problems. First, Equations (35) derive from the wave Equation (33) only

\footnotetext{
${ }^{2}$ This object, where $\boldsymbol{E}$ and $\boldsymbol{B}$ are the electric and magnetic fields, is known under the name of the Riemann-Silberstein wave function. Let us remind however, that this object has been introduced by these authors $[13,14]$, long before the advent of quantum mechanics.
} 
if the fields $\boldsymbol{E}$ and $\boldsymbol{B}$ are taken real. This is incompatible with the principle of the invariance of the wave function under a global phase shift, if the electromagnetic fields $\boldsymbol{E}$ and $\boldsymbol{B}$ have a physical reality, because they are changed by this phase shift. Second, the two circular polarization plane waves solutions require two different identifications of the components of their wave function $\psi$ (one with $\boldsymbol{E}$ and one with $\boldsymbol{B}$ as the real part of $\psi)$. Finally, the wave function $\psi=\boldsymbol{E}+i \boldsymbol{B}$ does not have the right properties under space inversion, if the real components are considered as physical electromagnetic fields. Indeed, electric fields change sign, whereas magnetic fields do not, and the Hamiltonian in Equation (4) commutes with the parity operator.

These difficulties do not appear in our formulation. If the 3-vectors $\Phi_{1}$ and $\Phi_{2}$ are identified with the electric and magnetic fields, respectively, the Equation (14) above are also identical to the Maxwell equations for free transverse fields. There is however an important difference in the two approaches. The functions $\Phi_{1}$ and $\Phi_{2}$ need not to be real. If they need to be related to physical fields, it is sufficient to consider the latter as the real (or imaginary) part of $\Phi_{1}$ and $\Phi_{2}$, as it is customary in the harmonic representation of classical electromagnetism. Applying a global phase shift to the wave function (9) merely corresponds to introducing a constant phase shift in the physical fields (at least for plane waves) and the physical reality attached to these fields is preserved. Furthermore, the plane wave solutions exhibit automatically the two helicities in our formulation, without the identification of the components with electric and magnetic fields. Finally, our wave function (9) has the right properties under space inversion, as described in Section 4.

These considerations are the main results of this note and show that if the wave function is to be related with physical electric and magnetic fields, the Raymer and Schmidt formulation (Equations $(33,34)$ ) does not seem to be consistent with quantum mechanics, contrarily to our formulation.

Let us also mention that the duality of the electromagnetic equations, namely that the equations are invariant under the substitution $\boldsymbol{E} \rightarrow \boldsymbol{B}, \boldsymbol{B} \rightarrow-\boldsymbol{E}$ is fulfilled by both our wave equation and the RaymerSchmidt one. The mere inspection of Equation (10) shows that they are invariant under the substitution $\Phi_{1} \rightarrow \Phi_{2}$, $\Phi_{2} \rightarrow-\Phi_{1}$. As for the wave Equation (33), the duality is evident from Equation (35), but is obtained by considering $\psi$ and $i \psi$ as solutions, if the real and imaginary parts of these wave functions are identified with physical electric and magnetic fields, respectively.

Finally, let us discuss a little bit the normalization of the wave function, since it interferes with the identifica- tion of the wave function with electromagnetic fields. Let us start with the probability density, which is given by Equation (11) in our formalism, provided the wave function is properly normalized as

$$
\int\left(\left|\Phi_{1}\right|^{2}+\left|\Phi_{2}\right|^{2}\right) \mathrm{d}^{3} \boldsymbol{r}=1
$$

The direct identification of $\Phi_{1}$ to $\boldsymbol{E}$ (and of $\Phi_{2}$ to $\boldsymbol{B}$ ) is not possible since these quantities do not have the same dimension. If $\Phi_{1}$ and $\Phi_{2}$ are to be related with physical electromagnetic fields, at least the identification mentioned above should be corrected by a constant factor. Indeed, the energy of the photon in state $\psi$ (Equation (9)) can be identified to the energy of the corresponding electromagnetic field. One should then require:

$$
\frac{c}{8 \pi} \int\left(\left|\Phi_{1}\right|^{2}+\left|\Phi_{2}\right|^{2}\right) \mathrm{d}^{3} \boldsymbol{r}=\langle H\rangle
$$

where the rhs is the quantum average of the Hamiltonian. This is a conserved quantity for a free photon. It can also be written as

$$
\langle H\rangle=-i \hbar\left\langle\psi \mid \frac{\partial}{\partial t} \psi\right\rangle .
$$

Therefore it may be more appropriate to make the identification

$$
\Phi_{1} \rightarrow \sqrt{\frac{c}{8 \pi}} \frac{\boldsymbol{E}}{\sqrt{\langle H\rangle}}, \Phi_{2} \rightarrow \sqrt{\frac{c}{8 \pi}} \frac{\boldsymbol{B}}{\sqrt{\langle H\rangle}} .
$$

The same considerations apply to the Raymer and Schmidt formulation.

There are however arguments against the interpretation of the components of the wave function as connected to the physical electric and magnetic fields attached to the photon. First of all, any physical quantity attached to the (free) photon should be associated to a Hermitian operator. But the only operators available in the quantum mechanics of a photon are linked with the translational and spin degrees of freedom and have no relation with electromagnetic properties. Strictly speaking, the electromagnetic field quantum mechanical operators are defined in the Fock space of field theory and have no effect in the Hilbert space of a single photon (like there is no operator linked to the electric field of the electron in the quantum mechanics of a single electron). Actually, the average value of the electric field or the magnetic field is vanishing. In face of these considerations, one may wonder whether the consistency between the photon wave equation and the Maxwell equations should not be interpreted differently, considering the components of the wave function as merely behaving as classical electromagnetic fields. They fulfill the same 
Maxwell equations, transform in the same way under Lorentz transformations (see below) and their equations are invariant under dual transformations. In other words, they may simply be objects with the same mathematical properties as the classical electromagnetic fields, but devoid of physical electromagnetic properties. They, of course, keep their physical meaning concerning the quantum probability density.

Within this new interpretation, some of the criticisms to the wave Equation (4) disappear. Let us consider a specific solution $\psi$. According to the previous interpretation, attaching some physical meaning to the real and imaginary part, the solution $\psi e^{i \phi}$ where $\phi$ is a real constant, is not equivalent to $\psi$, since the components are changed. On the contrary, in the new interpretation, the real and imaginary parts of the wave function have no physical meaning, but just behave as classical electromagnetic fields, both $\psi$ and $\psi e^{i \phi}$ are acceptable solutions of Equation (33). They however correspond to the same quantum mechanical reality (probability density and current).

\section{Invariance under Transformations of the Hamiltonian}

The wave Equation (10) acting in the space of divergenceless $\Phi_{1}$ and $\Phi_{2}$ has a special property: there are infinite numbers of Hamiltonians equivalent to the one of Equation (10), i.e. having the same solutions. Indeed it suffices to add any term generating the divergence of $\Phi_{1}$ and/or $\Phi_{2}$. For instance the substitution

$$
\left(\begin{array}{cc}
0 & -c \boldsymbol{p} \cdot \boldsymbol{H} \\
c \boldsymbol{p} \cdot \boldsymbol{H} & 0
\end{array}\right) \rightarrow\left(\begin{array}{cccc}
a p_{1} & a p_{2} & a p_{3} & \\
0 & 0 & 0 & -c \boldsymbol{p} \cdot \boldsymbol{H} \\
0 & 0 & 0 & \\
& c \boldsymbol{p} \cdot \boldsymbol{H} & 0
\end{array}\right)
$$

where $a$ is an arbitrary constant, generates an equivalent Hamiltonian. The transformed Hamiltonian does not look Hermitian, but since the line containing the $p$-operators gives a vanishing contribution in the space of divergenceless $\Phi_{1}$ and $\Phi_{2}$, this Hamiltonian is automatically Hermitian. It has exactly the same matrix elements as the original Hamiltonian in the space of divergenceless ${ }^{3} \Phi_{1}$ and $\Phi_{2}$. In fact, one can add a similar set of operators to any line of any of the four submatrices to generate equivalent Hamiltonians. Of course, it is much easier to drop all terms generating a divergence of $\Phi_{1}$ or $\Phi_{2}$. Nevertheless, this property will be used to discuss the Lorentz invariance in the next Section. Generalizing the argument, the Hamiltonians

\footnotetext{
${ }^{3}$ Note however that one has to be careful when applying the Hamiltonian on the bras; then one has to use the Hermitian conjugate of the $6 \times 6$ matrix in Equation (65).
}

$$
H^{\prime}=H+\sum_{k=1}^{6} a_{k} \sum_{i=1}^{3} p_{i} U_{k i}+\sum_{k=1}^{6} b_{k} \sum_{i=4}^{6} p_{i-3} U_{k i},
$$

where the $\boldsymbol{U}_{\boldsymbol{i} \boldsymbol{k}}$ are the $6 \times 6$ matrices defined in Equation (66), are equivalent to the original Hamiltonian, i.e. they have the same eigenvalues and the same (divergenceless) eigenfunctions.

\section{Lorentz Invariance}

\subsection{Introductory Remark}

The question of the Lorentz invariance of the wave equation (10) may appear as a trivial issue, since the components $\Phi_{1}$ and $\Phi_{2}$ behave as classical electromagnetic fields. Some points however need a clarification. First, Equation (10) is restricted to divergenceless functions and the condition for vanishing divergence has no obvious Lorentz covariance property. Second, $\Phi_{1}$ and $\Phi_{2}$ are associated in a 6-vector and not in a tensor like $F_{\mu \nu}$, which is the usual basis to discuss Lorentz invariance. It would then be desirable to prove explicitly the Lorentz invariance of the wave equation. Here below, we restrict ourselves to show explicitly the Lorentz invariance for a Lorentz boost and for space inversion, following closely the method ordinarily used for the Dirac equation.

\subsection{Lorentz Invariance for a Boost}

We start with the remark that the wave Equation (10) can be cast, after multiplication on the left by the a nonsingular $\gamma^{0}$ matrix, in the following Dirac form (for a massless particle)

$$
\gamma^{\mu} \partial_{\mu} \psi=0
$$

with

$$
\gamma^{i}=\boldsymbol{\gamma}^{0}\left(\begin{array}{cc}
0 & -H_{i} \\
H_{i} & 0
\end{array}\right)
$$

Note that these gamma matrices are $6 \times 6$ matrices. The $\gamma^{0}$ matrix is arbritrary, except that its square should be equal to the identity matrix. It is tempting to take

$$
\boldsymbol{\gamma}^{0}=\left(\begin{array}{cc}
\underline{1} & 0 \\
0 & -\underline{1}
\end{array}\right) \text {. }
$$

The $\gamma^{i}$ matrices are then given by

$$
\gamma^{i}=\left(\begin{array}{cc}
0 & -H_{i} \\
-H_{i} & 0
\end{array}\right) .
$$

Using these matrices and momentum operators, the wave Equation (42) can be written as: 


$$
\left(\begin{array}{cc}
p_{0} \underline{1} & -c \boldsymbol{p} \cdot \boldsymbol{H} \\
-c \boldsymbol{p} \cdot \boldsymbol{H} & p_{0} \underline{1}
\end{array}\right)\left(\begin{array}{l}
\Phi_{1} \\
\boldsymbol{\Phi}_{2}
\end{array}\right)=0,
$$

which, with $p_{0}=(\hbar / i) \partial / \partial t$, is equivalent to Equation (10). The gamma matrices introduced here do not follow the same anticommutation relations as the Dirac matrices. The reason is that the square of the operator in Equation (42) is not equal to $p^{2}$, but to an operator which reduces to $p^{2}$ for divergenceless functions. Presumably, the gamma matrices (43) are not unique and Equation (42) is, like the Dirac equation, independant of the representation. We did not investigate this point.

We shall not attempt to derive the Lorentz invariance in general. Following the method described in Reference [15], we will verify this property for one particular transformation, namely a boost along the $z$ axis. According to this reference, it is sufficient to show that there exists a matrix $S$, relating the wave functions in the two different frames by $\psi^{\prime}=\boldsymbol{S} \psi$, which is such that if $\psi$ is a solution of Equation (42), $\psi^{\prime}$ is solution of the wave equation written in the transformed frame:

$$
\gamma^{\mu} \partial_{u}^{\prime} \psi^{\prime}=0
$$

This equation may be rewritten as

$$
\gamma^{\mu} a_{\mu}^{v} \partial_{v} S \psi=0
$$

where $a_{\mu}^{v}$ is the Lorentz transformation matrix. Following the usual method, it is thus sufficient to show that there exists a matrix $\boldsymbol{S}$ satisfying

$$
\gamma^{\mu} a_{\mu}^{v}=\boldsymbol{S} \gamma^{v} .
$$

However, according to the discussion of the preceding Section, one must admit in the right hand side of Equation (48), additional terms, which give a vanishing contribution for divergenceless $\Phi_{1}$ and $\Phi_{2}$, and the previous condition becomes

$$
\gamma^{\mu} a_{\mu}^{v} \boldsymbol{S} \partial_{v}=\gamma^{v} \partial_{v}+\cdots
$$

where the dots stand for such terms, and any other terms giving vanishing contributions in the wave equation.

We merely show that the following matrix

$$
\boldsymbol{S}=\left(\begin{array}{ccccc}
\gamma & & & & \\
& \gamma & & & H_{3} \beta \gamma \\
& & 1 & & \\
& & \gamma & & \\
-H_{3} \beta \gamma & & & \gamma & \\
& & & & 1
\end{array}\right)
$$

satisfies these requirements. In this equation, $-\beta$ is the velocity of the primed frame with respect to the unprimed one. The corresponding Lorentz transformation matrix in Equation (50) is given by

$$
\boldsymbol{a}=\left(\begin{array}{cccc}
\gamma & 0 & 0 & \beta \gamma \\
0 & 1 & 0 & 0 \\
0 & 0 & 1 & 0 \\
\beta \gamma & 0 & 0 & \gamma
\end{array}\right)
$$

We leave the detail of the calculation in Appendix C. We collect the results for the operator of the lhs of Equation (48) from Equations (75-78):

$$
\begin{aligned}
\gamma^{\mu} a_{\mu}^{v} p_{v} S & =\gamma^{v} p_{v}-\beta \gamma\left(U_{64} p_{1}+U_{65} p_{2}+U_{66} p_{3}\right) \\
& +\beta \gamma\left(U_{31} p_{1}+U_{32} p_{2}+U_{33} p_{3}\right) \\
& +(\gamma-1)\left(U_{33} p_{0}-U_{35} p_{1}+U_{34} p_{2}\right) \\
& +(\gamma-1)\left(-U_{66} p_{0}-U_{62} p_{1}+U_{61} p_{2}\right)
\end{aligned}
$$

where the $6 \times 6$ matrices $\boldsymbol{U}_{\boldsymbol{a}}$ have all vanishing elements except for the one at the crossing of line $a$ and column $b$, which is equal to one. It is then very easy to see that the second and third terms of the rhs of the last equation gives vanishing contributions when applied to divergenceless $\Phi_{1}$ and $\Phi_{2}$. It can also easily be seen that the terms proportional to $(\gamma-1)$ are simply the z-components of the two Equations (14), giving thus also a vanishing contribution. One then recovers the Equations (46) or the Equations (14), except that the third and sixth equations are multiplied by $(\gamma-1)$.

There is no secret beyond the matrix (51). It is nothing but the matrix expressing the transformation of classical transverse electromagnetic fields form one frame to the other:

$$
\left(\begin{array}{l}
\boldsymbol{E}^{\prime} \\
\boldsymbol{B}^{\prime}
\end{array}\right)=S\left(\begin{array}{l}
\boldsymbol{E} \\
\boldsymbol{B}
\end{array}\right),
$$

see [16]. However, we consider here wave functions and thus we have to take care of some quantum mechanical requirements. First, Equation (10) acts on divergenceless functions. Therefore, one has to verify that this transformation preserves the vanishing divergence of the transformed components $\Phi_{1}$ and $\Phi_{2}$. Once again, we limit the demonstration to the Lorentz boost described by Equation (52). In this case, one has, with

$$
\begin{aligned}
\Phi_{1}= & \left(\left(\Phi_{1}\right)_{1},\left(\Phi_{1}\right)_{2}\left(\Phi_{1}\right)_{3}\right), \Phi_{2}=\left(\left(\Phi_{2}\right)_{1},\left(\Phi_{2}\right)_{2}\left(\Phi_{2}\right)_{3}\right): \\
& \partial_{1}^{\prime}\left(\Phi_{1}^{\prime}\right)_{1}+\partial_{2}^{\prime}\left(\Phi_{1}^{\prime}\right)_{2}+\partial_{3}^{\prime}\left(\Phi_{1}^{\prime}\right)_{3} \\
= & \partial_{1}\left(\Phi_{1}^{\prime}\right)_{1}+\partial_{2}\left(\Phi_{1}^{\prime}\right)_{2}+\beta \gamma \partial_{t}\left(\Phi_{1}^{\prime}\right)_{3}+\gamma \partial_{3}\left(\Phi_{1}^{\prime}\right)_{3} \\
= & \partial_{t}\left(\gamma\left(\Phi_{1}\right)_{1}-\beta \gamma\left(\Phi_{2}\right)_{2}\right)+\partial_{2}\left(\gamma\left(\Phi_{1}\right)_{2}+\beta \gamma\left(\Phi_{2}\right)_{1}\right) \\
& +\beta \gamma \partial_{t}\left(\Phi_{1}\right)_{3}+\gamma \partial_{3}\left(\Phi_{1}\right)_{3} \\
= & \gamma\left(\partial_{1}\left(\Phi_{1}\right)_{1}+\partial_{2}\left(\Phi_{1}\right)_{2}+\partial_{3}\left(\Phi_{1}\right)_{3}\right) \\
& +\beta \gamma\left(-\partial_{1}\left(\Phi_{2}\right)_{2}+\partial_{2}\left(\Phi_{2}\right)_{1}+\partial_{t}\left(\Phi_{2}\right)_{3}\right)
\end{aligned}
$$

In the second line, the explicit form of $\boldsymbol{S}$ (Equation 
(51)) has been used. The last parenthesis vanishes, by virtue of Equation (35) or Equation (14). This shows that $\Phi_{1}$ is divergenceless if $\Phi_{1}$ is divergenceless. The similar property for $\Phi_{2}$ is obtained on exactly the same way. Note that this does not mean that any divergenceless function in a frame is automatically divergenceless in any other frame. In the derivation of Equation (55), explicit use of the wave Equation (10) (or the Maxwell equations), linking $\Phi_{1}$ and $\Phi_{2}$, has been made.

Let us notice that the transformation $\psi^{\prime}=\boldsymbol{S} \psi$, with $\boldsymbol{S}$ given by Equation (51) is not a unitary transformation and therefore does not conserve the norm of the wave function. This, of course, is consistent with the fact that, for a classical electromagnetic field $(\boldsymbol{E}, \boldsymbol{B})$, the quantity $\left(E_{3}\right)^{2}+\left(B_{3}\right)^{2}$ is an invariant, whereas its integral (over coordinate $\boldsymbol{r}$ in one case and coordinate $\boldsymbol{r}^{\prime}$ in the other case) is not invariant under a Lorentz transformation (along the z-direction). In other words, if one has normalized the wave function as in Equation (36) in a given frame, the wave function transformed through $\psi^{\prime}=\boldsymbol{S} \psi \quad$ (with $\boldsymbol{S}$ given by Equation (51)) in another frame does not automatically fulfill Equation (36) in this new frame.

\subsection{Lorentz Invariance for Space Inversion}

It is interesting to consider the Lorentz transformation corresponding to space inversion. Again, we have to find a matrix $S_{P}$ which satisfies ${ }^{4}$

$$
\gamma^{\mu} a_{\mu}^{v} \boldsymbol{S}_{\boldsymbol{P}}=\boldsymbol{S}_{\boldsymbol{P}} \gamma^{v}
$$

where $a_{\mu}^{v}$ is

$$
\boldsymbol{a}=\left(\begin{array}{cccc}
1 & 0 & 0 & 0 \\
0 & -1 & 0 & 0 \\
0 & 0 & -1 & 0 \\
0 & 0 & 0 & -1
\end{array}\right)
$$

It is easy to see that $S_{P}$ is the $6 \times 6$ matrix

$$
\boldsymbol{S}_{P}=\left(\begin{array}{cc}
1 & 0 \\
0 & -1
\end{array}\right)
$$

We get the same result as for the Dirac equation (except that in the latter case $S_{P}$ is a $4 \times 4$ matrix). Here also, there is no surprise, since our Equation (42) has the same structure as the Dirac equation. Since the wave function is behaving like an electromagnetic wave, space inversion leaves $\Phi_{1}$ unchanged and flips the sign of $\Phi_{2}$.

\footnotetext{
${ }^{4}$ Since the wave Equation (42) is homogeneous and linear in the gamma matrices, one can require in general that $\gamma^{\mu} a_{\mu}^{v} \boldsymbol{S}=\boldsymbol{A} \gamma^{v}$, where

$\boldsymbol{A}$ is a non-singular matrix: $\boldsymbol{A} \gamma^{\mu} \partial_{\mu} \psi=0$ then implies $\gamma^{\mu} \partial_{\mu} \psi=0$. For the invariance under a Lorentz boost, discussed earlier, the matrix $\boldsymbol{A}$ has been implicitly taken equal to the unit matrix.
}

\section{Conclusions}

We have re-analyzed the properties of a wave equation for a six-component wave function of a photon. This wave equation and this wave function have been already proposed in the past by several authors. The purpose of this work was a careful analysis of the quantum and invariance properties of the formalism. We have shown that the properties of the latter are more consistent with the principles of quantum mechanics than those of the three-component wave equation and wave function proposed in Reference [12]. We have shown that the difficulty for the latter choice comes from the interpretation of the wave function as an observable electromagnetic field. If this interpretation is abandoned, the inconsistency of the formalism of Reference [12] with quantum mechanics disappears, except for the problems which are linked with the position operator, that survive in our formulation as well. Let us however mention that the three-component wave equation does not admit plane wave solutions $\psi=\boldsymbol{a} e^{i(\boldsymbol{k} \cdot \boldsymbol{r}-\omega t)}$ with a 3 -vector $\boldsymbol{a}$ for real $\omega^{5}$. That is the reason why the introduction of polarization is not natural in the wave Equation (4) or (33).

We have also demonstrated explicitly the Lorentz invariance of the six-component wave equation for Lorentz boosts and space inversion.

Let us finally mention that the results are rather transparent for free photon solutions in vacuum. As underlined in [3], the real interest of the formalism lies in the treatment of the propagation of photons in media.

\section{Acknowledgments}

We are very thankful to Dr. Jean-René Cudell for interesting discussions and a careful reading of the manuscript.

\section{References}

[1] V. V. Dvoeglazov, "2(2S + 1)-Component Model and Its Connection with Other Field Theories," XVII Symposium on Nuclear Physics, Mexico, 4-7 January 1994.

[2] I. Bialynicki-Birula, "On the Wave Function of the Photon," Acta Physica Polonica A, Vol. 86, 1994, pp. 97116.

[3] I. Bialynicki-Birula, "Photon Wave Function," In: E. Wolf Ed., Progress in Optics XXXVI, Elsevier, Amsterdam, 1996.

[4] I. Bialynicki-Birula, "Exponential Localization of Photons," Physical Review Letters, Vol. 80, No. 24, 1998, pp.

${ }^{5}$ This property is mathematically consistent with the fact that plane wave solutions of the Maxwell equations correspond to electric and magnetic components with a phase shift of 90 degrees. 
5247-5250. doi:10.1103/PhysRevLett.80.5247

[5] J. E. Sipe, "Photon Wave Functions," Physical Review A, Vol. 52, No. 3, 1995, pp. 1875-1883. doi:10.1103/PhysRevLett.80.5247

[6] D. H. Kobe, "A Relativistic Schrödinger-Like Equation for a Photon and Its Second Quantization," Foundations of Physics, Vol. 29, No. 8, 1999, pp. 1203-1231. doi:10.1023/A:1018855630724

[7] M. Hawton, "Lorentz-Invariant Photon Number Density," Physical Review A, Vol. 78, No. 1, 2008, Article ID 012111. doi:10.1103/PhysRevA.78.012111

[8] H. Feshbach and F. Villars, "Elementary Relativistic Wave Mechanics of Spin 0 and Spin 1/2 Particles," Reviews of Modern Physics, Vol. 30, No. 1, 1958, pp. 2445. doi:10.1103/RevModPhys.30.24

[9] Z.-Y. Wang, C.-D. Xiong and O. Keller, "The FirstQuantized Theory of Photons," Chinese Physics Letters, Vol. 24, No. 2, 2007, pp. 418-420. doi:10.1088/0256-307X/24/2/032

[10] Z.-Y. Wang, C.-D. Xiong and O. Keller, "Photon Wave Mechanics," Chinese Physics Letters, Vol. 24, No. 2, 2007, arXiv:quant-ph/0511181v5.
[11] S. Esposito, E. Recami, A. Van der Merwe and R. Battiston, "Ettore Majorana: Unpublished Research Notes on Theoretical Physics," Springer, Berlin, 2009. doi:10.1007/978-1-4020-9114-8

[12] M. G. Raymer and B. J. Smith, "The Maxwell Wave Function of the Photon," SPIE Conference Optics and Photonics, San Diego, August 2005.

[13] H. Weber, "Notes on Differential Equations by Riemann," In: Riemann and G. F. Berhard, Eds., Die Partiellen Differential-Gleichungen der Mathematischen Physik Nach Riemann's Vorlesungen, Friedrich Vieweg und Sohn, Brunswick, 1901, p. 348.

[14] L. Silberstein, "Elektromagnetische Grundgleichungen in Bivectorieller Behandlung," Annalen der Physik, Vol. 22, 1907, p. 579. doi:10.1002/andp.19073270313

[15] J. J. Sakurai, “Advanced Quantum Mechanics," AddisonWesley Publication Co., London, 1967.

[16] J. D. Jackson, "Classical Electrodynamics," John Wiley, New York, 1975, pp. 552-554. 


\section{Appendix A: Properties of the $\boldsymbol{H}_{i}$ Matrices}

The matrices

$$
\boldsymbol{H}_{1}=\left(\begin{array}{ccc}
0 & 0 & 0 \\
0 & 0 & -1 \\
0 & 1 & 0
\end{array}\right), \boldsymbol{H}_{2}=\left(\begin{array}{ccc}
0 & 0 & 1 \\
0 & 0 & 0 \\
-1 & 0 & 0
\end{array}\right), \boldsymbol{H}_{3}=\left(\begin{array}{ccc}
0 & -1 & 0 \\
1 & 0 & 0 \\
0 & 0 & 0
\end{array}\right)
$$

possess the following property:

$$
\left(\boldsymbol{H}_{i}\right)_{j k}=-\varepsilon_{i j k}
$$

Therefore, the scalar product of an arbitrary vector $\boldsymbol{a}$ with $\boldsymbol{H}$ is a $3 \times 3$ matrix, whose matrix elements are given by

$$
(\boldsymbol{a} \cdot \boldsymbol{H})_{j k}=-\sum_{l} \varepsilon_{l j k} \boldsymbol{a}_{l}
$$

If this matrix multiplies a column vector $\boldsymbol{b}$, the $j$-th element of the resulting column vector is given by

$$
((\boldsymbol{a} \cdot \boldsymbol{H}) \cdot \boldsymbol{b})_{j}=\sum_{l} \sum_{k} \varepsilon_{j l k} a_{l} b_{k}=(\boldsymbol{a} \times \boldsymbol{b})_{j} .
$$

One can thus write

$$
(\boldsymbol{a} \cdot \boldsymbol{H}) \cdot \boldsymbol{b}=\boldsymbol{a} \times \boldsymbol{b} .
$$

The square of $(\boldsymbol{a} \cdot \boldsymbol{H})$ is a $3 \times 3$ matrix, whose elements are given by

$$
\begin{aligned}
& \left((\boldsymbol{a} \cdot \boldsymbol{H})^{2}\right)_{j k}=\sum_{m}(\boldsymbol{a} \cdot \boldsymbol{H})_{j m}(\boldsymbol{a} \cdot \boldsymbol{H})_{m k} \\
& =\sum_{l} a_{l} \varepsilon_{l j m} \sum_{l^{\prime}} a_{l^{\prime}} \varepsilon_{l j m}=-\delta_{j k} \boldsymbol{a}^{2}+a_{j} a_{k} .
\end{aligned}
$$

The matrices $\boldsymbol{H}_{i}$ have also some other remarkable properties. The product of two of them $\boldsymbol{H}_{i} \boldsymbol{H}_{j}$, for $i \neq j$ is given by

$$
\left(\boldsymbol{H}_{i} \boldsymbol{H}_{j}\right)_{k l}=\left(U_{i j}\right)_{k l}=\delta_{i k} \delta_{j l} .
$$

i.e., for instance, $\boldsymbol{H}_{1} \boldsymbol{H}_{2}$ has all vanishing elements, but the element $(1,2)$, which is equal to one. Note that, in this paper, the matrices $\boldsymbol{U}_{i j}$ are defined by

$$
\left(\boldsymbol{U}_{i j}\right)_{k l}=\delta_{i k} \delta_{j l} \text {. }
$$

even for $i=j$. The squares of the matrices $\boldsymbol{H}_{\boldsymbol{i}}$ are given by

$$
\boldsymbol{H}_{i}^{2}=-\underline{-1}_{i}
$$

where $\underline{1}_{i}$ is the unit $3 \times 3$ matrix in which the i-th diagonal element has been replaced by 0 . More generally,

$$
\boldsymbol{H}_{i}^{4 n+1}=\boldsymbol{H}_{i}, \boldsymbol{H}_{i}^{4 n+2}=-\underline{1}_{i}, \boldsymbol{H}_{i}^{4 n+3}=-\boldsymbol{H}_{i}, \boldsymbol{H}_{i}^{4 n}=\underline{1}_{i},
$$

and

$$
\boldsymbol{H} \cdot \boldsymbol{H}=-2 \times \underline{1} .
$$

Properties (65) and (67)are summarized formally in

$$
\left(\boldsymbol{H}_{i} \boldsymbol{H}_{j}\right)_{k l}=\sum_{m} \varepsilon_{i k m} \varepsilon_{j m l}=-\delta_{i j} \delta_{k l}+\delta_{i k} \delta_{j l}
$$

Finally, the matrices $\boldsymbol{H}_{\boldsymbol{i}}$ satisfy the following commutation relations

$$
\left[\boldsymbol{H}_{i}, \boldsymbol{H}_{j}\right]=-\varepsilon_{i j k} \boldsymbol{H}_{k} .
$$

Thus, the matrices $i \boldsymbol{H}_{j}, j=1,2,3$, are the adjoint representation of the $\mathrm{SU}(3)$ algebra.

\section{Appendix B: Probability Density and Current}

Starting with Equation (14), multiying the first line on the left by $\Phi_{1}^{*}$, and the second line by $\Phi_{2}^{*}$ and summing, one readily gets

$$
\begin{aligned}
& \Phi_{1}^{*} \frac{\partial}{\partial t} \Phi_{1}+\Phi_{2}^{*} \frac{\partial}{\partial t} \vec{\Phi}_{2} \\
& =c \Phi_{1}^{*} \cdot\left(\nabla \times \Phi_{2}\right)-c \Phi_{2}^{*} \cdot\left(\nabla \times \Phi_{1}\right) .
\end{aligned}
$$

Summing this equation and its complex conjugate leads to

$$
\begin{aligned}
\frac{\partial}{\partial t}\left(\left|\Phi_{1}\right|^{2}+\left|\Phi_{2}\right|^{2}\right)= & c \Phi_{1}^{*} \cdot\left(\nabla \times \Phi_{2}\right)-c \Phi_{2}^{*} \cdot\left(\nabla \times \Phi_{1}\right) \\
& +c \Phi_{1} \cdot\left(\nabla \times \Phi_{2}^{*}\right)-c \Phi_{2} \cdot\left(\nabla \times \Phi_{1}^{*}\right)
\end{aligned}
$$

Using $\nabla \cdot(\boldsymbol{a} \times \boldsymbol{b})=\boldsymbol{b} \cdot(\nabla \times \boldsymbol{a})-\boldsymbol{a} \cdot(\nabla \times \boldsymbol{b})$, one readily gets

$$
\frac{\partial}{\partial t}\left(\left|\Phi_{1}\right|^{2}+\left|\Phi_{2}\right|^{2}\right)=\nabla \cdot\left(c \Phi_{1}^{*} \times \Phi_{2}+c \Phi_{2}^{*} \times \Phi_{1}\right) .
$$

\section{Appendix C: Demonstration of Equation (53)}

Let us consider the left hand side of Equation (50) for $v=$ 0 . Using Equations (52) and (51), one has 


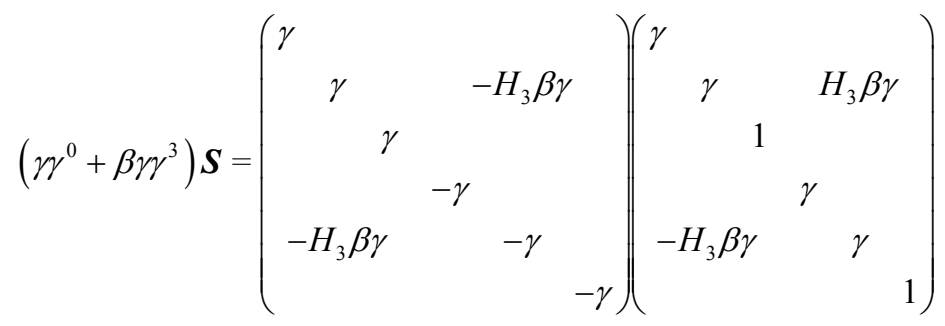

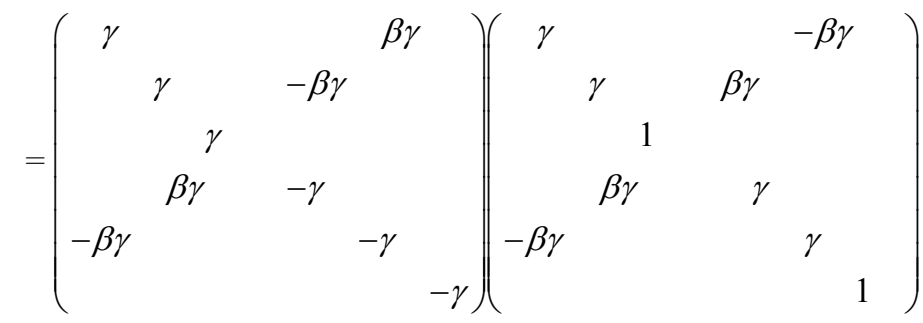

$$
\begin{aligned}
& =\left(\begin{array}{llllll}
1 & & & & & \\
& 1 & & & & \\
& & \gamma & & & \\
& & -1 & & \\
& & & -1 & \\
& & & & & \gamma
\end{array}\right) \\
& =\gamma^{0}+(\gamma-1) U_{33}-(\gamma-1) U_{66},
\end{aligned}
$$

where $U_{i j}$ is the $6 \times 6$ matrix defined in Equation (66), ing of line $i$ and column $j$, which is equal to one. whose elements are vanishing, except the one at the cross-

For $v=1,2$, one has successively

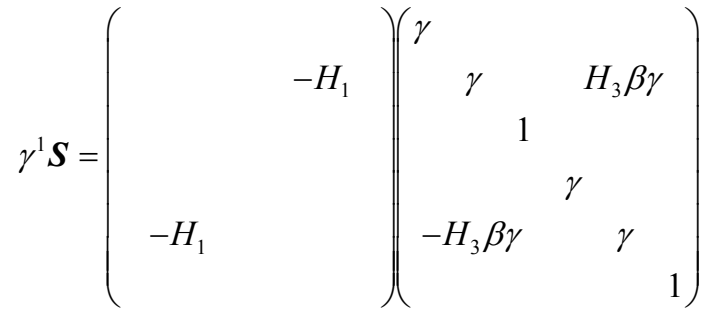

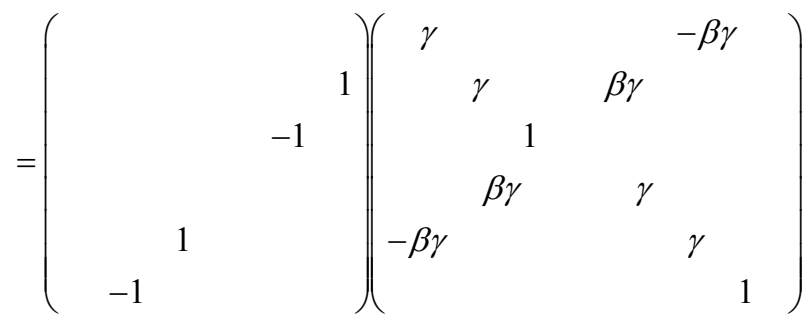

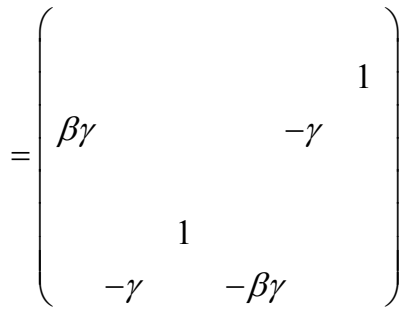

$$
\begin{aligned}
& =\gamma^{1}+U_{31} \beta \gamma-U_{64} \beta \gamma-(\gamma-1) U_{35}-(\gamma-1) U_{62},
\end{aligned}
$$




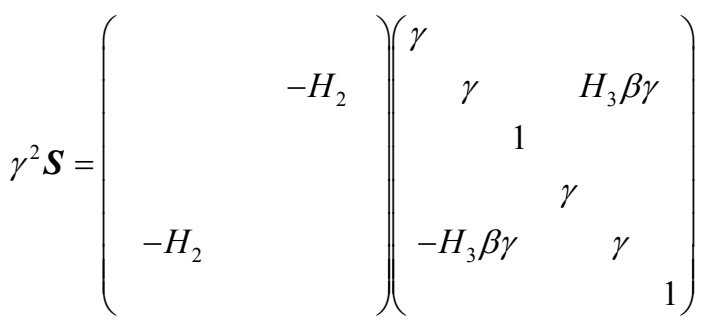

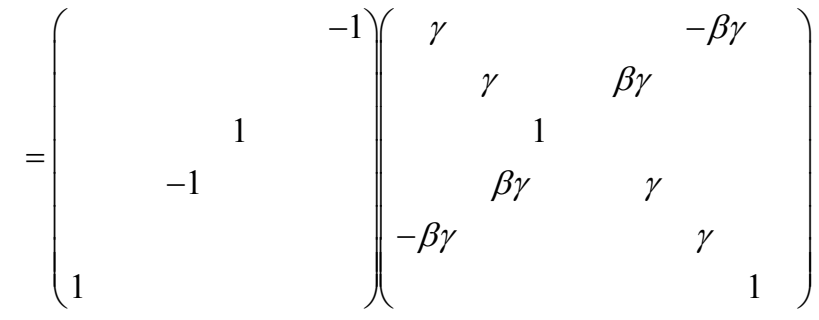

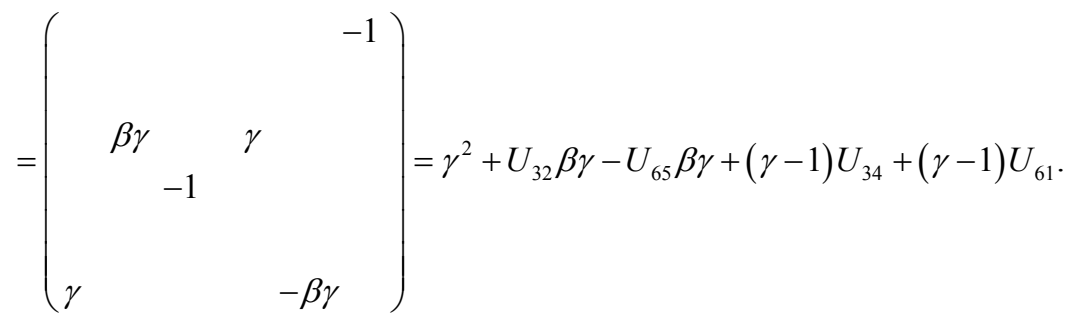

Finally, one has, for $v=3$,

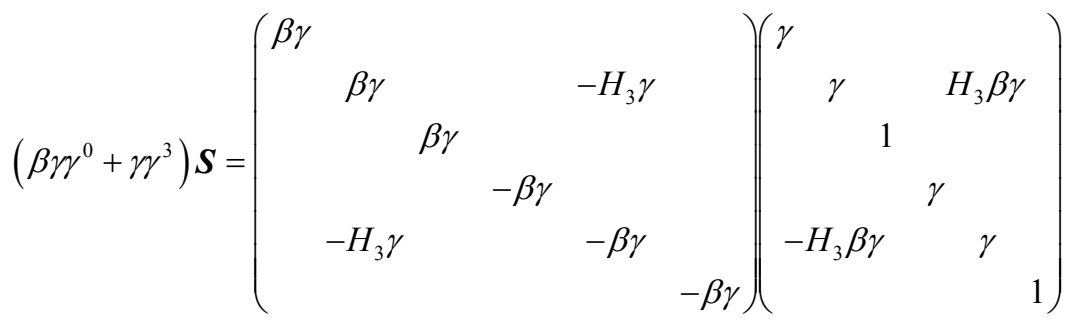

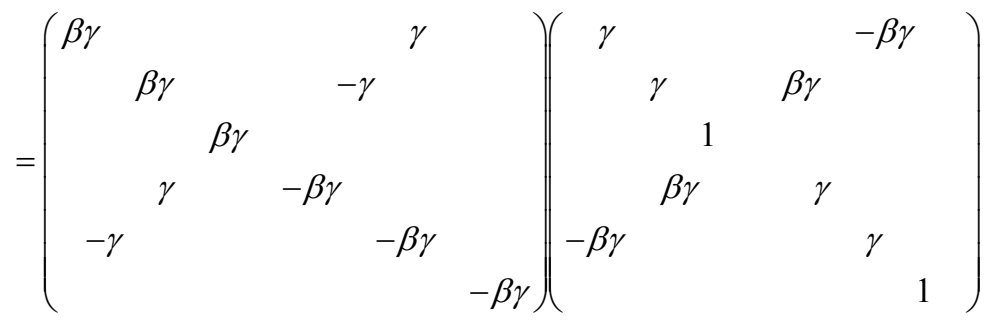

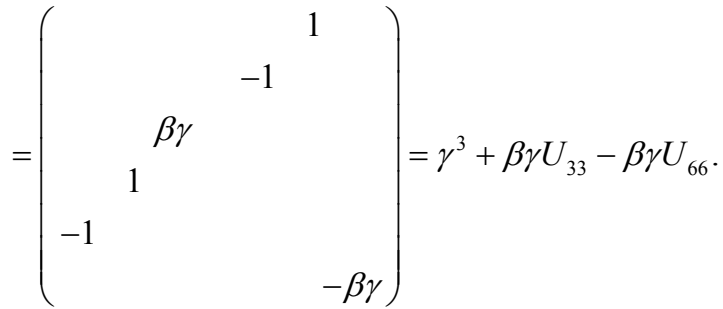

